\title{
A NOTE ON HYPONORMAL WEIGHTED SHIFTS
}

\author{
PENG FAN
}

Dedicated to the memory of my sister Jing (1958-1983)

\begin{abstract}
If $T$ is a hyponormal weighted shift (unilateral or bilateral) and if $p$ is a polynomial, $p(T)$ may not be hyponormal.
\end{abstract}

If $T$ is a hyponormal shift (unilateral or bilateral) and if $p$ is a polynomial, $p(T)$ may not be hyponormal. This answers negatively Question 33 of Shields [1], initially raised by Hong Wha Kim. The question is: If $T$ is a hyponormal unilateral shift and if $p$ is a polynomial, must $p(T)$ be hyponormal?

In what follows, we shall first construct a hyponormal bilateral shift $T$ and a polynomial $p$ so that $p(T)$ is not hyponormal. Then, by compressing $T$ to a proper subspace, we shall show that the resulting hyponormal unilateral shift $S$ also possesses the property that $p(S)$ is not hyponormal.

Let $T$ be a bilateral shift defined by

$$
T e_{n}= \begin{cases}e_{n+1} & \text { for } n \leqslant 2, \\ 2 e_{n+1} & \text { for } n \geqslant 3,\end{cases}
$$

and let $p(z)=z+a z^{2}$ for $0<a<\sqrt{5} / 5$. Since the weight sequence of $T$ is nondecreasing, $T$ is hyponormal. Next, an elementary computation shows that

$$
\left[p(T)^{*}, p(T)\right] \quad\left(=p(T)^{*} p(T)-p(T) p(T)^{*}\right)=0_{1} \oplus A \oplus 0_{2},
$$

where $0_{1}$ and $0_{2}$ are zero operators defined on $H_{1}=V_{n \leqslant 1}\left\{e_{n}\right\}$ (the subspace spanned by $\left.\left\{e_{n}\right\}_{n \leqslant 1}\right)$ and $H_{2}=V_{n=5}^{\infty}\left\{e_{n}\right\}$, respectively, and

$$
A=\left[\begin{array}{ccc}
3 a^{2} & 3 a & 0 \\
3 a & 3+15 a^{2} & 6 a \\
0 & 6 a & 12 a^{2}
\end{array}\right]
$$

is the matrix representation of the compression of $\left[p(T)^{*}, p(T)\right]$ to $V\left\{e_{2}, e_{3}, e_{4}\right\}$ with respect to basis $\left\{e_{2}, e_{3}, e_{4}\right\}$. Since Det $A=108 a^{4}\left(5 a^{2}-1\right)<0,\left[p(T)^{*}, p(T)\right]$ is not positive. Therefore, $p(T)$ is not hyponormal as desired.

Now, let $S$ be the compression of $T$ to $H_{0}=V_{n=0}^{\infty}\left\{e_{n}\right\}$. That is, $S=\left.P_{H_{0}} T\right|_{H_{0}}$ in which $P_{H_{0}}$ denotes the projection operator onto $H_{0}$. Clearly, $S$ is a hyponormal

Received by the editors April 28, 1983.

1980 Mathematics Subject Classification. Primary 47B20, 47B37. 
unilateral shift. An almost identical computation provides us with

$$
\left[p(S)^{*}, P(S)\right]=B \oplus A \oplus 0_{2}
$$

where $A$ and $\mathrm{O}_{2}$ are defined as above and

$$
B=\left[\begin{array}{cc}
1+a^{2} & a \\
a & a^{2}
\end{array}\right]
$$

is the matrix representation of the compression of $\left[p(S)^{*}, P(S)\right]$ to $V\left\{e_{0}, e_{1}\right\}$ with respect to basis $\left\{e_{0}, e_{1}\right\}$. An identical argument concludes the proof that $p(S)$ is not hyponormal.

\section{REFERENCES}

1. A. L. Shields, Weighted shift operators and analytic function theory, Math. Surveys, no. 13, Amer. Math. Soc., Providence, R. I., 1974.

Department of Mathematics, Marshall University, Huntington, West Virginia 25701 